Research Article

\title{
Structural Dynamics Model Updating with Positive Definiteness and No Spillover
}

\author{
Yongxin Yuan \\ School of Mathematics and Statistics, Hubei Normal University, Huangshi 435002, China \\ Correspondence should be addressed to Yongxin Yuan; yuanyx_703@163.com
}

Received 21 February 2014; Revised 16 May 2014; Accepted 22 May 2014; Published 3 June 2014

Academic Editor: Mohammad I. Younis

Copyright (C) 2014 Yongxin Yuan. This is an open access article distributed under the Creative Commons Attribution License, which permits unrestricted use, distribution, and reproduction in any medium, provided the original work is properly cited.

Model updating is a common method to improve the correlation between structural dynamics models and measured data. In conducting the updating, it is desirable to match only the measured spectral data without tampering with the other unmeasured and unknown eigeninformation in the original model (if so, the model is said to be updated with no spillover) and to maintain the positive definiteness of the coefficient matrices. In this paper, an efficient numerical method for updating mass and stiffness matrices simultaneously is presented. The method first updates the modal frequencies. Then, a method is presented to construct a transformation matrix and this matrix is used to correct the analytical eigenvectors so that the updated model is compatible with the measurement of the eigenvectors. The method can preserve both no spillover and the symmetric positive definiteness of the mass and stiffness matrices. The method is computationally efficient as neither iteration nor numerical optimization is required. The numerical example shows that the presented method is quite accurate and efficient.

\section{Introduction}

Using finite element techniques, the undamped free vibration of a structural dynamics system can be described by the second order differential equation as

$$
M_{a} \ddot{q}(t)+K_{a} q(t)=f(t),
$$

where $M_{a}, K_{a} \in \mathbf{R}^{n \times n}$ are analytical mass and stiffness matrices, $q(t)$ is the $n \times 1$ vector of positions, and $f(t)$ is the $n \times 1$ vector of external force. In many practical applications, $M_{a}$ and $K_{a}$ are real-valued symmetric and positive definite, denoted by $M_{a}>0$ and $K_{a}>0$. Equation (1) is usually known as the finite element analytical model. By considering the homogeneous part of (1) and assuming that the displacement response of (1) is harmonic,

$$
q(t)=x(\omega) e^{i \omega t}
$$

then the structural eigenproblem can be written in the form:

$$
K_{a} x_{j}=\lambda_{j} M_{a} x_{j}, \quad j=1,2, \ldots, n,
$$

where $\lambda_{j}=\omega_{j}^{2}$ is the $j$ th eigenvalue and $x_{j}$ is the $j$ th eigenvector. It is well known that the eigenvalue and eigenvector can be interpreted physically as the square of the natural frequency of vibration and the mode shape, respectively. Let

$$
\Lambda=\left[\begin{array}{cc}
\Lambda_{1} & 0 \\
0 & \Lambda_{2}
\end{array}\right], \quad X=\left[X_{1}, X_{2}\right],
$$

where $\Lambda_{1}=\operatorname{diag}\left\{\lambda_{1}, \ldots, \lambda_{p}\right\}, \Lambda_{2}=\operatorname{diag}\left\{\lambda_{p+1}, \ldots, \lambda_{n}\right\}, X_{1}=$ $\left[x_{1}, \ldots, x_{p}\right]$, and $X_{2}=\left[x_{p+1}, \ldots, x_{n}\right]$. Then it is easy to see that the $n$ columns of the matrix

$$
M_{a} X \Lambda=K_{a} X
$$

summarise $n$ separate eigenvalue-eigenvector relations of type (3). The most important property of the undamped vibration modes is their orthogonality with respect to mass; that is,

$$
\left[\begin{array}{l}
X_{1}^{\top} \\
X_{2}^{\top}
\end{array}\right] M_{a}\left[X_{1}, X_{2}\right]=I_{n} .
$$


Substituting (6) into (5), we can obtain another orthogonality relation

$$
\left[\begin{array}{c}
X_{1}^{\top} \\
X_{2}^{\top}
\end{array}\right] K_{a}\left[X_{1}, X_{2}\right]=\Lambda .
$$

Accurate finite element models are needed for a large number of applications such as validating innovative structural designs, evaluating the effects of earthquakes or strong winds on in-service structures, and the implementation of structural control and structural health monitoring strategies. But there are some inaccuracies or uncertainties that may be associated with a finite element model. The discretization error, arising due to the approximation of a continuous structure by a finite number of individual elements, is inherent to the finite element technique, while other inaccuracies may be due to the assumptions and simplifications made by the analyst with regard to the choice of elements, modelling of boundary conditions, joints, and so forth. Therefore, once a finite element model of a structural system is constructed, its accuracy must be tested by comparing its analytical modes of vibration (or natural frequencies and mode shapes) with those obtained from the structural system during a modal survey. If the agreement between the two is good, then more credence is given to the analytical model, and it can be used with confidence for future analysis. If the correlation between the two is poor, then, assuming that the experimental measurements are correct, the analytical model must be adjusted so that the agreement between predictions and test results is improved. The updated model may then be considered to be a better dynamical representation of the structure than the initial analytical model. The updated model can subsequently be used with reasonable accuracy to assess the stability and control characteristics and to predict the dynamical responses of the structure. The above process of correcting the system matrices is known as model updating.

In general, the measurements will be incomplete in the sense that the measurement frequency range (determined by the sampling rate) will be much shorter than that of the analytical model which might typically contain several thousands of degrees of freedom. The measurements will also be spatially incomplete. This arises because the number of measurement stations is generally very much smaller than the number of degrees of freedom in the finite element model. For example, rotational degrees of freedom are usually not measured and some degrees of freedom will be inaccessible. Thus, a few eigenvalues and the corresponding eigenvectors measured only at some of the degrees of freedom can be used to adjust the analytical model parameters.

On the other hand, in conducting the updating, it is often desirable to match only the part of observed data without tampering with the other part of unmeasured or unknown eigenstructure inherent in the original model. Such an updating is said to be of no spillover. In this case assume that the matrices of part spectral information $\Lambda_{1} \in \mathbf{R}^{p \times p}$, $X_{1} \in \mathbf{R}^{n \times p}$ are known for the first $p$ eigenvalues and associated eigenvectors of the original system. The remainder of the spectral properties $\Lambda_{2} \in \mathbf{R}^{(n-p) \times(n-p)}, X_{2} \in \mathbf{R}^{n \times(n-p)}$ are unknown and should not be changed. No spillover is required either because these high modal data $\left(X_{2}, \Lambda_{2}\right)$ are proven to be acceptable in the original model and engineers do not wish to introduce new vibrations via updating or because engineers simply do not know any information about these modal data.

In the past 40 years a large number of model updating methods have been developed and discussed in the literature surveys carried out by Mottershead and Friswell $[1,2]$. In the early 1980s, Lagrange multiplier methods were introduced by Baruch and Bar-Itzhack [3] and Berman and Nagy [4]. These methods usually assumed that the test mode shapes or/and the mass matrix are correct. Then an objective function, with constraints imposed through Lagrange multipliers, is minimised in order to derive updated system matrices. The matrix mixing methods were developed by Caesar [5] and Link et al. [6]. This approach sought to combine experimental modal data with analytical ones to construct the inverses of the mass and stiffness matrices. The control-based eigenstructure assignment techniques were proposed by Zimmerman and Widengren [7] and Inman and Minas [8]. These early methods have the merits of improving the analytical structural dynamic models and are computationally efficient. However, these methods do not preserve the updated mass and stiffness matrices are symmetric positive definite and cannot guarantee that the updated model is of no spillover. Recently, assuming that the mass matrix is exact, Carvalho et al. [9] proposed a direct method for undamped model updating with no spillover. Chu et al. [10-12] considered damped model updating with no spillover. The purpose of the work presented in this paper is to develop a new direct method which can preserve both no spillover and the positive definiteness of the updated mass and stiffness matrices. Compared with the method proposed by Mao and Dai [13], we observe that the approach provided by this paper is more simple and easy to perform. The problem of updating mass and stiffness matrices simultaneously can be mathematically formulated as follows.

Problem $P$. Assume that only the part $Y_{11}$ of the experimental eigenvector matrix $Y_{1}=\left[\begin{array}{l}Y_{11} \\ Y_{12}\end{array}\right] \in \mathbf{R}^{n \times p}$ is measured and $Y_{12}$ is unknown, where $Y_{11} \in \mathbf{R}^{m \times p}$ and $\operatorname{rank}\left(Y_{11}\right)=p$. Let $\Gamma_{1}=\operatorname{diag}\left\{\gamma_{1}, \ldots, \gamma_{p}\right\} \in \mathbf{R}^{p \times p}>0$ be the measured eigenvalue matrix. Find real-valued symmetric positive definite matrices $M$ and $K$ such that

$$
M Y_{1} \Gamma_{1}=K Y_{1}, \quad M X_{2} \Lambda_{2}=K X_{2}
$$

The paper is organized as follows. In Section 2, a method is provided to handle the problem of incomplete experimental mode shapes and the incomplete part (i.e., $Y_{12}$ ) of the experimental modes which cannot be measured is supplied by means of an effective numerical procedure. In Section 3, an efficient numerical method for solving Problem $\mathrm{P}$ is presented. The method first updates the modal frequencies. Then, a method is presented to construct a transformation matrix $S$. This matrix is used to correct the analytical eigenvectors so that the updated model is compatible with the measurement of the eigenvectors. In Section 4, a numerical 
algorithm to acquire the solution of Problem $\mathrm{P}$ is described and a numerical example is provided. Some concluding remarks are given in Section 5.

\section{Expansion of the Measured Mode Shapes}

Lemma 1 (see $[11,14]$ ). The equation of (8) has a symmetric solution pair $(M, K)$ if and only if there exists an invertible matrix $L \in \mathbf{R}^{p \times p}$ such that

$$
Y_{1}=X_{1} L
$$

Now, if one lets

$$
X_{1}=\left[\begin{array}{l}
X_{11} \\
X_{12}
\end{array}\right]
$$

where $X_{11} \in \mathbf{R}^{m \times p}$, then from (9) one has

$$
Y_{11}=X_{11} L, \quad Y_{12}=X_{12} L .
$$

As the approach in [14], let the QR factorization of $X_{11}$ be

$$
X_{11}=\left[Q_{1}, Q_{2}\right]\left[\begin{array}{l}
R \\
0
\end{array}\right],
$$

where $Q$ is an $m \times m$ orthogonal matrix and $R \in \mathbf{R}^{p \times p}$ is an upper triangular matrix. The condition $\operatorname{rank}\left(Y_{11}\right)=p$ implies that $R$ is nonsingular. Substituting (12) into the first equation of (11), we have

$$
\begin{gathered}
Q_{2}^{\top} Y_{11}=0, \\
R L=Q_{1}^{\top} Y_{11} .
\end{gathered}
$$

Hence, if the relation of (13) is satisfied, then (14) has a unique solution $L=R^{-1} Q_{1}^{\top} Y_{11}$. Substituting this relation into the second equation of (11), we have

$$
Y_{12}=X_{12} R^{-1} Q_{1}^{\top} Y_{11} \text {. }
$$

Thus, the eigenvector matrix $Y$ with full degrees of freedom can be computed:

$$
Y_{1}=X_{1} R^{-1} Q_{1}^{\top} Y_{11}
$$

\section{The Solution of Problem P}

Applying (6) and (7), we have

$$
\begin{gathered}
M_{a}^{-1}=X_{1} X_{1}^{\top}+X_{2} X_{2}^{\top}, \\
M_{a}^{-1} K_{a} M_{a}^{-1}=X_{1} \Lambda_{1} X_{1}^{\top}+X_{2} \Lambda_{2} X_{2}^{\top} .
\end{gathered}
$$

Define the matrix $\widetilde{K}$ as

$$
M_{a}^{-1} \widetilde{K} M_{a}^{-1}=X_{1} \Gamma_{1} X_{1}^{\top}+X_{2} \Lambda_{2} X_{2}^{\top} .
$$

From (17) and (18), we obtain

$$
\widetilde{K}=K_{a}+M_{a} X_{1}\left(\Gamma_{1}-\Lambda_{1}\right) X_{1}^{\top} M_{a} .
$$

Using (5), (6), and (19), we have

$$
\begin{aligned}
\widetilde{K} X= & K_{a} X+M_{a} X_{1}\left(\Gamma_{1}-\Lambda_{1}\right) X_{1}^{\top} M_{a} X \\
= & M_{a}\left[X_{1}, X_{2}\right]\left[\begin{array}{cc}
\Lambda_{1} & 0 \\
0 & \Lambda_{2}
\end{array}\right] \\
& +M_{a} X_{1}\left(\Gamma_{1}-\Lambda_{1}\right) X_{1}^{\top} M_{a}\left[X_{1}, X_{2}\right]
\end{aligned}
$$

$$
\begin{aligned}
& =M_{a}\left[X_{1}, X_{2}\right]\left[\begin{array}{cc}
\Lambda_{1} & 0 \\
0 & \Lambda_{2}
\end{array}\right]+M_{a}\left[X_{1}, X_{2}\right]\left[\begin{array}{cc}
\Gamma_{1}-\Lambda_{1} & 0 \\
0 & 0
\end{array}\right] \\
& =M_{a}\left[X_{1}, X_{2}\right]\left[\begin{array}{cc}
\Gamma_{1} & 0 \\
0 & \Lambda_{2}
\end{array}\right]=M_{a} X\left[\begin{array}{cc}
\Gamma_{1} & 0 \\
0 & \Lambda_{2}
\end{array}\right],
\end{aligned}
$$

which implies that the model $\left(M_{a}, \widetilde{K}\right)$ possesses the eigenvalues $\gamma_{1}, \ldots, \gamma_{p}, \lambda_{p+1}, \ldots, \lambda_{n}$. It follows from $\Gamma_{1}=$ $\operatorname{diag}\left\{\gamma_{1}, \ldots, \gamma_{p}\right\}>0$ that the matrix $\widetilde{K}$, defined by (19), is symmetric positive definite. If we can find a nonsingular transformation matrix $S$ such that

$$
S\left[X_{1}, X_{2}\right]=\left[Y_{1}, X_{2}\right]
$$

then define matrices $M, K$ as

$$
\begin{gathered}
M=\left(S^{-1}\right)^{\top} M_{a} S^{-1}, \\
K=\left(S^{-1}\right)^{\top} \widetilde{K} S^{-1}=\left(S^{-1}\right)^{\top} K_{a} S^{-1} \\
+\left(S^{-1}\right)^{\top} M_{a} X_{1}\left(\Gamma_{1}-\Lambda_{1}\right) X_{1}^{\top} M_{a} S^{-1} .
\end{gathered}
$$

It can be easily seen that $M$ and $K$ are symmetric positive definite matrices. From (21), (22), we can obtain

$$
\begin{aligned}
K\left[Y_{1}, X_{2}\right] & =\left(S^{-1}\right)^{\top} \widetilde{K} S^{-1}\left[Y_{1}, X_{2}\right] \\
& =\left(S^{-1}\right)^{\top} \widetilde{K}\left[X_{1}, X_{2}\right] \\
& =\left(S^{-1}\right)^{\top} M_{a}\left[X_{1}, X_{2}\right]\left[\begin{array}{cc}
\Gamma_{1} & 0 \\
0 & \Lambda_{2}
\end{array}\right] \\
& =\left(S^{-1}\right)^{\top} M_{a} S^{-1}\left[Y_{1}, X_{2}\right]\left[\begin{array}{cc}
\Gamma_{1} & 0 \\
0 & \Lambda_{2}
\end{array}\right] \\
& =M\left[Y_{1}, X_{2}\right]\left[\begin{array}{cc}
\Gamma_{1} & 0 \\
0 & \Lambda_{2}
\end{array}\right],
\end{aligned}
$$

which implies that $M$ and $K$, defined by (22), are a solution of Problem P. From (21), (22), we also have

$$
\left[Y_{1}, X_{2}\right]^{\top} M\left[Y_{1}, X_{2}\right]=(S X)^{\top}\left(\left(S^{-1}\right)^{\top} M_{a} S^{-1}\right) S X=I_{n} ;
$$

that is, the orthogonality with respect to mass is also satisfied.

The following theorem will provide a sufficient and necessary condition for the existence of the nonsingular transformation matrix $S$.

Theorem 2. There exists a nonsingular transformation matrix $S$ such that the relation (21) holds if and only if $X_{1}^{\top} M_{a} Y_{1}$ is nonsingular.

Proof. Because $X=\left[X_{1}, X_{2}\right]$ is nonsingular, the column vectors of $X$ form a basis of $\mathbf{R}^{n}$. Therefore, the column vectors of $Y_{1}$ can be expressed as the unique linear combination of those of $X$. That is,

$$
Y_{1}=\left[X_{1}, X_{2}\right] Z
$$


for some matrix $Z \in \mathbf{R}^{n \times p}$. Let

$$
Z=\left[\begin{array}{c}
Z_{1} \\
Z_{2}
\end{array}\right], \quad Z_{1} \in \mathbf{R}^{m \times m}
$$

Then the relation of (25) is equivalent to

$$
Y_{1}=X_{1} Z_{1}+X_{2} Z_{2}
$$

By (21), we can easily see that $S$ is nonsingular if and only if $\left[Y_{1}, X_{2}\right]$ is nonsingular. It follows from (27) that

$$
\left[Y_{1}, X_{2}\right]=\left[X_{1} Z_{1}+X_{2} Z_{2}, X_{2}\right]=\left[X_{1}, X_{2}\right]\left[\begin{array}{cc}
Z_{1} & 0 \\
Z_{2} & I_{n-m}
\end{array}\right]
$$

Therefore, $\left[Y_{1}, X_{2}\right]$ is nonsingular if and only if $Z_{1}$ is nonsingular. We observe that

$$
X_{1}^{\top} M_{a} Y_{1}=X_{1}^{\top} M_{a}\left(X_{1} Z_{1}+X_{2} Z_{2}\right)=Z_{1} .
$$

So, $S$ is nonsingular if and only if $X_{1}^{\top} M_{a} Y_{1}$ is nonsingular. This proves Theorem 2.

Now, we are ready to solve $S$. By (21) and (17), we have

$$
\begin{aligned}
S\left[X_{1}, X_{2}\right]\left[\begin{array}{c}
X_{1}^{\top} \\
X_{2}^{\top}
\end{array}\right]=\left[Y_{1}, X_{2}\right]\left[\begin{array}{c}
X_{1}^{\top} \\
X_{2}^{\top}
\end{array}\right] \\
\Longleftrightarrow S M_{a}^{-1}=Y_{1} X_{1}^{\top}+M_{a}^{-1}-X_{1} X_{1}^{\top} ;
\end{aligned}
$$

then it follows that

$$
S=I_{n}+\left(Y_{1}-X_{1}\right) X_{1}^{\top} M_{a}
$$

Combining Theorem 2 with the relation (16), we have the following corollary.

Corollary 3. Problem $P$ is solvable if and only if condition (13) is satisfied. In this case, the solution of Problem $P$ is given by (22), where $S$ is given by (31).

In practice, the matrices $X_{2}$ and $\Lambda_{2}$ are usually unknown. Now, we construct the solution of Problem $\mathrm{P}$ without using $X_{2}$ and $\Lambda_{2}$ explicitly. We should also point out that the method proposed in this paper is similar to that in [15]. That is, the framework is the same and both include three main steps: expansion of eigenvectors, updating of eigenvalues, and updating of eigenvectors. The differences between [15] and the current paper are eigenvector expansion techniques and the method of computing transformation matrix $S$ in (21).

\section{A Numerical Example}

Based on Corollary 3 we can describe an algorithm for solving Problem $\mathrm{P}$ as follows.

Algorithm 4.

(1) Input $M_{a}, K_{a}, \Lambda_{1}, X_{1}, \Gamma_{1}, Y_{11}$.

(2) Partition $X_{1}$ as $X_{1}=\left[\begin{array}{l}X_{11} \\ X_{12}\end{array}\right]$, and let the $\mathrm{QR}$ factorization of $X_{11}$ be given by (12).

(3) If condition (13) is satisfied, then continue; otherwise, Problem P has no solution; stop.

(4) Compute $Y_{1}$ by (16)

(5) Compute $S$ by (31).

(6) Compute the solution $(M, K)$ of Problem P by (22).

Example 1. Consider a 10-DOF cantilever beam modelled analytically with mass and stiffness matrices given by

$$
\begin{aligned}
M_{a} & =0.03 \\
& \times\left[\begin{array}{cccccccccc}
52 & 22 & 18 & -13 & 0 & 0 & 0 & 0 & 0 & 0 \\
22 & 12 & 13 & -9 & 0 & 0 & 0 & 0 & 0 & 0 \\
18 & 13 & 104 & 0 & 18 & -13 & 0 & 0 & 0 & 0 \\
-13 & -9 & 0 & 24 & 13 & -9 & 0 & 0 & 0 & 0 \\
0 & 0 & 18 & 13 & 104 & 0 & 18 & -13 & 0 & 0 \\
0 & 0 & -13 & -9 & 0 & 24 & 13 & -9 & 0 & 0 \\
0 & 0 & 0 & 0 & 18 & 13 & 104 & 0 & 18 & -13 \\
0 & 0 & 0 & 0 & -13 & -9 & 0 & 24 & 13 & -9 \\
0 & 0 & 0 & 0 & 0 & 0 & 18 & 13 & 104 & 0 \\
0 & 0 & 0 & 0 & 0 & 0 & -13 & -9 & 0 & 24
\end{array}\right], \\
K_{a} & =600 \times\left[\begin{array}{ccccccccccc}
2 & 3 & -2 & 3 & 0 & 0 & 0 & 0 & 0 & 0 \\
3 & 6 & -3 & 3 & 0 & 0 & 0 & 0 & 0 & 0 \\
-2 & -3 & 4 & 0 & -2 & 3 & 0 & 0 & 0 & 0 \\
3 & 3 & 0 & 12 & -3 & 3 & 0 & 0 & 0 & 0 \\
0 & 0 & -2 & -3 & 4 & 0 & -2 & 3 & 0 & 0 \\
0 & 0 & 3 & 3 & 0 & 12 & -3 & 3 & 0 & 0 \\
0 & 0 & 0 & 0 & -2 & -3 & 4 & 0 & -2 & 3 \\
0 & 0 & 0 & 0 & 3 & 3 & 0 & 12 & -3 & 3 \\
0 & 0 & 0 & 0 & 0 & 0 & -2 & -3 & 4 & 0 \\
0 & 0 & 0 & 0 & 0 & 0 & 3 & 3 & 0 & 12
\end{array}\right] .
\end{aligned}
$$

$\Lambda_{1}$ and $X_{1}$ are given by

$$
\begin{gathered}
\Lambda_{1}=\operatorname{diag}\{0.4710,18.5145,146.0550,569.9851\}, \\
X_{1}=\left[\begin{array}{cccc}
0.4364 & 0.4369 & -0.4394 & -0.4447 \\
-0.0401 & -0.1392 & 0.2301 & 0.3281 \\
0.3166 & 0.0306 & 0.1738 & 0.2881 \\
-0.0396 & -0.1249 & 0.1386 & 0.0522 \\
0.2013 & -0.2575 & 0.2081 & -0.1481 \\
-0.0367 & -0.0588 & -0.1112 & -0.2024 \\
0.1003 & -0.2986 & -0.2314 & -0.1400 \\
-0.0298 & 0.0295 & -0.1189 & 0.2081 \\
0.0279 & -0.1315 & -0.2657 & 0.3375 \\
-0.0176 & 0.0677 & 0.0914 & -0.0182
\end{array}\right] .
\end{gathered}
$$


The measured modal data are given by

$$
\begin{gathered}
\Gamma_{1}=\operatorname{diag}\{0.30142,14.626,122.69,493.04\}, \\
Y_{11}=\left[\begin{array}{cccc}
0.7431 & 1.5119 & 1.8188 & -0.0103 \\
-0.1872 & -0.2838 & -0.1720 & 1.4176 \\
0.1955 & 0.6780 & 1.2366 & 3.0038 \\
-0.1697 & -0.2619 & -0.2484 & 0.0591 \\
-0.2081 & 0.0259 & 0.2410 & 0.2760
\end{array}\right] .
\end{gathered}
$$

It is easy to check that $Q_{2}^{\top} Y_{11}=2.4808 e-016$. By (16), we can get

$$
\begin{aligned}
& M= {\left[\begin{array}{ccccccccccc}
1.7844 & 0.6679 & -0.6574 & -0.5215 & 0.8013 & 0.7375 & 2.8601 & -0.0497 & 1.2009 & -0.4243 \\
0.6679 & 0.3220 & -0.2341 & -0.3106 & 0.2620 & 0.3111 & 1.0989 & -0.0288 & 0.4190 & -0.1617 \\
-0.6574 & -0.2341 & 0.8738 & 0.2621 & 0.6148 & 0.0706 & 0.2538 & -0.3613 & -1.0379 & 0.0304 \\
-0.5215 & -0.3106 & 0.2621 & 0.8201 & 0.5613 & -0.4009 & -0.5642 & -0.1127 & -0.5506 & 0.1106 \\
0.8013 & 0.2620 & 0.6148 & 0.5613 & 7.4000 & 1.0031 & 4.7598 & -1.4315 & -0.6148 & -0.4095 \\
0.7375 & 0.3111 & 0.0706 & -0.4009 & 1.0031 & 0.9722 & 1.8612 & -0.3855 & 0.4586 & -0.1956 \\
2.8601 & 1.0989 & 0.2538 & -0.5642 & 4.7598 & 1.8612 & 8.8878 & -1.0219 & 0.5172 & -1.0491 \\
-0.0497 & -0.0288 & -0.3613 & -0.1127 & -1.4315 & -0.3855 & -1.0219 & 0.8450 & 0.1065 & -0.1481 \\
1.2009 & 0.4190 & -1.0379 & -0.5506 & -0.6148 & 0.4586 & 0.5172 & 0.1065 & 1.9446 & 0.0440 \\
-0.4243 & -0.1617 & 0.0304 & 0.1106 & -0.4095 & -0.1956 & -1.0491 & -0.1481 & 0.0440 & 0.7956
\end{array}\right], } \\
& K=1000 \times\left[\begin{array}{ccccccccccc}
1.1341 & 1.7848 & -1.0428 & 1.8284 & -0.0412 & -0.0681 & -0.0535 & 0.0647 & 0.1858 & -0.0039 \\
1.7848 & 3.5964 & -1.7637 & 1.8073 & -0.0027 & -0.0145 & -0.0071 & 0.0131 & 0.0409 & -0.0013 \\
-1.0428 & -1.7637 & 2.0411 & -0.0599 & -1.0332 & 1.9722 & 0.1999 & -0.1628 & -0.4232 & 0.0005 \\
1.8284 & 1.8073 & -0.0599 & 7.1867 & -1.7939 & 1.8258 & 0.0247 & -0.0200 & -0.0562 & -0.0002 \\
-0.0412 & -0.0027 & -1.0332 & -1.7939 & 2.2438 & -0.0812 & -1.2700 & 1.9100 & 0.2972 & -0.0112 \\
-0.0681 & -0.0145 & 1.9722 & 1.8258 & -0.0812 & 7.1195 & -1.8862 & 1.8795 & 0.2109 & -0.0020 \\
-0.0535 & -0.0071 & 0.1999 & 0.0247 & -1.2700 & -1.8862 & 2.2944 & 0.0745 & -1.0111 & 1.8020 \\
0.0647 & 0.0131 & -0.1628 & -0.0200 & 1.9100 & 1.8795 & 0.0745 & 7.1081 & -2.0490 & 1.8065 \\
0.1858 & 0.0409 & -0.4232 & -0.0562 & 0.2972 & 0.2109 & -1.0111 & -2.0490 & 1.7200 & 0.0196 \\
-0.0039 & -0.0013 & 0.0005 & -0.0002 & -0.0112 & -0.0020 & 1.8020 & 1.8065 & 0.0196 & 7.1984
\end{array}\right] .
\end{aligned}
$$

(36)

$$
Y_{1}=\left[\begin{array}{cccc}
0.7431 & 1.5119 & 1.8188 & -0.0103 \\
-0.1872 & -0.2838 & -0.1720 & 1.4176 \\
0.1955 & 0.6780 & 1.2366 & 3.0038 \\
-0.1697 & -0.2619 & -0.2484 & 0.0591 \\
-0.2081 & 0.0259 & 0.2410 & 0.2760 \\
-0.0890 & -0.1595 & -0.3878 & -1.1818 \\
-0.3080 & -0.2361 & -0.6995 & -0.1886 \\
0.0204 & -0.0164 & -0.1644 & 0.8774 \\
-0.1438 & -0.1366 & -0.5581 & 1.8034 \\
0.0724 & 0.0637 & 0.2216 & -0.1779
\end{array}\right] \text {. }
$$

Using Algorithm 4, we obtain the solution of Problem P as follows:
It is easy to check that

$$
\begin{gathered}
M>0, \quad K>0, \\
\left\|K Y_{1}-M Y_{1} \Gamma_{1}\right\|=9.6144 e-012, \\
\left\|Y_{1}^{\top} M Y_{1}-I_{4}\right\|=6.6485 e-015, \\
\left\|Y_{1}^{\top} K Y_{1}-\Gamma_{1}\right\|=7.3741 e-012 .
\end{gathered}
$$

Therefore, the prescribed eigenvalues (the diagonal elements of the matrix $\Gamma_{1}$ ) and eigenvectors (the column vectors of the matrix $Y_{1}$ ) are embedded in the new model $M Y_{1} \Gamma_{1}=$ $K Y_{1}$, and the mass normalized orthogonality constraints are also satisfied. Furthermore, we observe that the remaining 
symmetric positive definite. The proposed method is computationally efficient since it does not require computation of the complete set of eigenvalues and eigenvectors of the analytical model. The approach is demonstrated by a 10-DOF cantilever beam numerical example and reasonable results are produced.

\section{Conflict of Interests}

The author declares that there is no conflict of interests regarding the publication of this paper.

\section{Acknowledgment}

The author would like to express his heartfelt thanks to the anonymous reviewers whose constructive criticisms and helpful suggestions were very useful in the preparation of the current revised version of the paper.

\section{References}

[1] J. E. Mottershead and M. I. Friswell, "Model updating in structural dynamics: a survey," Journal of Sound and Vibration, vol. 167, no. 2, pp. 347-375, 1993.

[2] M. I. Friswell and J. E. Mottershead, Finite Element Model Updating in Structural Dynamics, vol. 38 of Solid Mechanics and Its Applications, Kluwer Academic Publishers, Dordrecht, The Netherlands, 1995.

[3] M. Baruch and I. Y. Bar-Itzhack, "Optimal weighted orthogonalization of measured modes," AIAA Journal, vol. 16, no. 4, pp. 346-351, 1978.

[4] A. Berman and E. J. Nagy, "Improvement of a large analytical model using test data," AIAA Journal, vol. 21, no. 8, pp. 1168$1173,1983$.

[5] B. Caesar, "Updating system matrices using modal testing data," in Proceedings of the 5th International Modal Analysis Conference (IMAC '87), pp. 453-459, London, UK, 1987.

[6] M. Link, M. Weiland, and J. M. Barragan, "Direct physical matrix identification as compared to phase resonance testing: assessment based on practical application," in Proceedings of the 5th International Modal Analysis Conference (IMAC '87), pp. 804-811, London, UK, 1987.

[7] D. Zimmerman and M. Widengren, "Correcting finite element models using a symmetric eigenstructure assignment technique," AIAA Journal, vol. 28, no. 9, pp. 1670-1676, 1990.

[8] D. J. Inman and C. Minas, "Matching analytical models with experimental modal data in mechanical systems," Control and Dynamics of Systems, vol. 37, pp. 327-363, 1990.

[9] J. Carvalho, B. N. Datta, A. Gupta, and M. Lagadapati, "A direct method for model updating with incomplete measured data and without spurious modes," Mechanical Systems and Signal Processing, vol. 21, no. 7, pp. 2715-2731, 2007.

[10] M. T. Chu, W.-W. Lin, and S.-F. Xu, "Updating quadratic models with no spillover effect on unmeasured spectral data," Inverse Problems, vol. 23, no. 1, pp. 243-256, 2007.

[11] M. T. Chu, B. Datta, W.-W. Lin, and S.-F. Xu, "Spillover phenomenon in quadratic model updating," AIAA Journal, vol. 46, no. 2, pp. 420-428, 2008.

[12] D. Chu, M. T. Chu, and W.-W. Lin, "Quadratic model updating with symmetry, positive definiteness, and no spill-over," SIAM
Journal on Matrix Analysis and Applications, vol. 31, no. 2, pp. 546-564, 2009.

[13] X. Mao and H. Dai, "Finite element model updating with positive definiteness and no spill-over," Mechanical Systems and Signal Processing, vol. 28, pp. 387-398, 2012.

[14] Y.-C. Kuo and B. N. Datta, "Quadratic model updating with no spill-over and incomplete measured data: existence and computation of solution," Linear Algebra and its Applications, vol. 436, no. 7, pp. 2480-2493, 2012.

[15] K.-V. Yuen, "Updating large models for mechanical systems using incomplete modal measurement," Mechanical Systems and Signal Processing, vol. 28, pp. 297-308, 2012. 


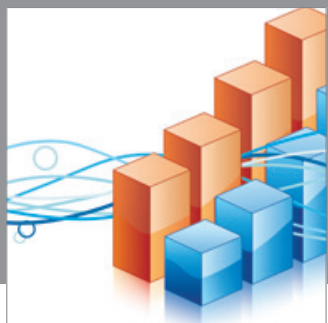

Advances in

Operations Research

mansans

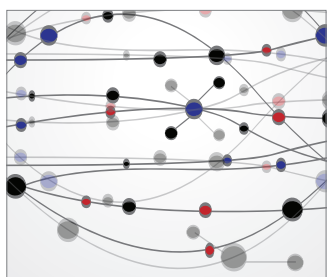

The Scientific World Journal
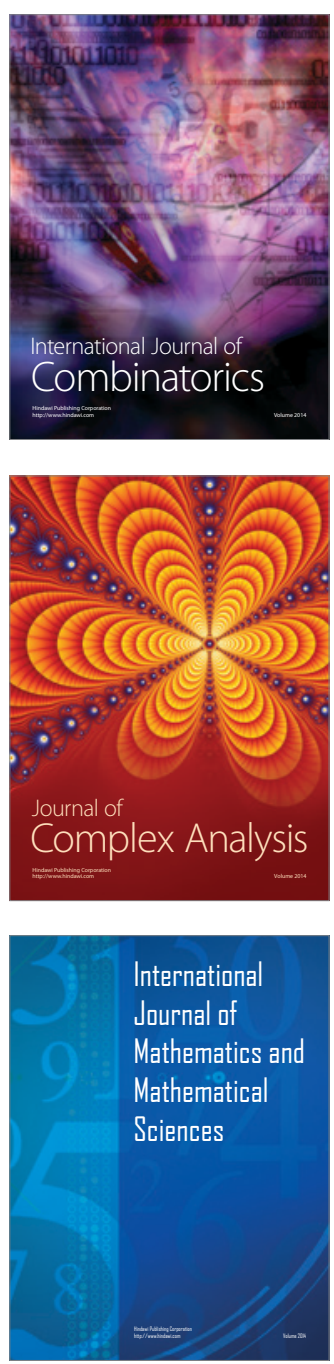
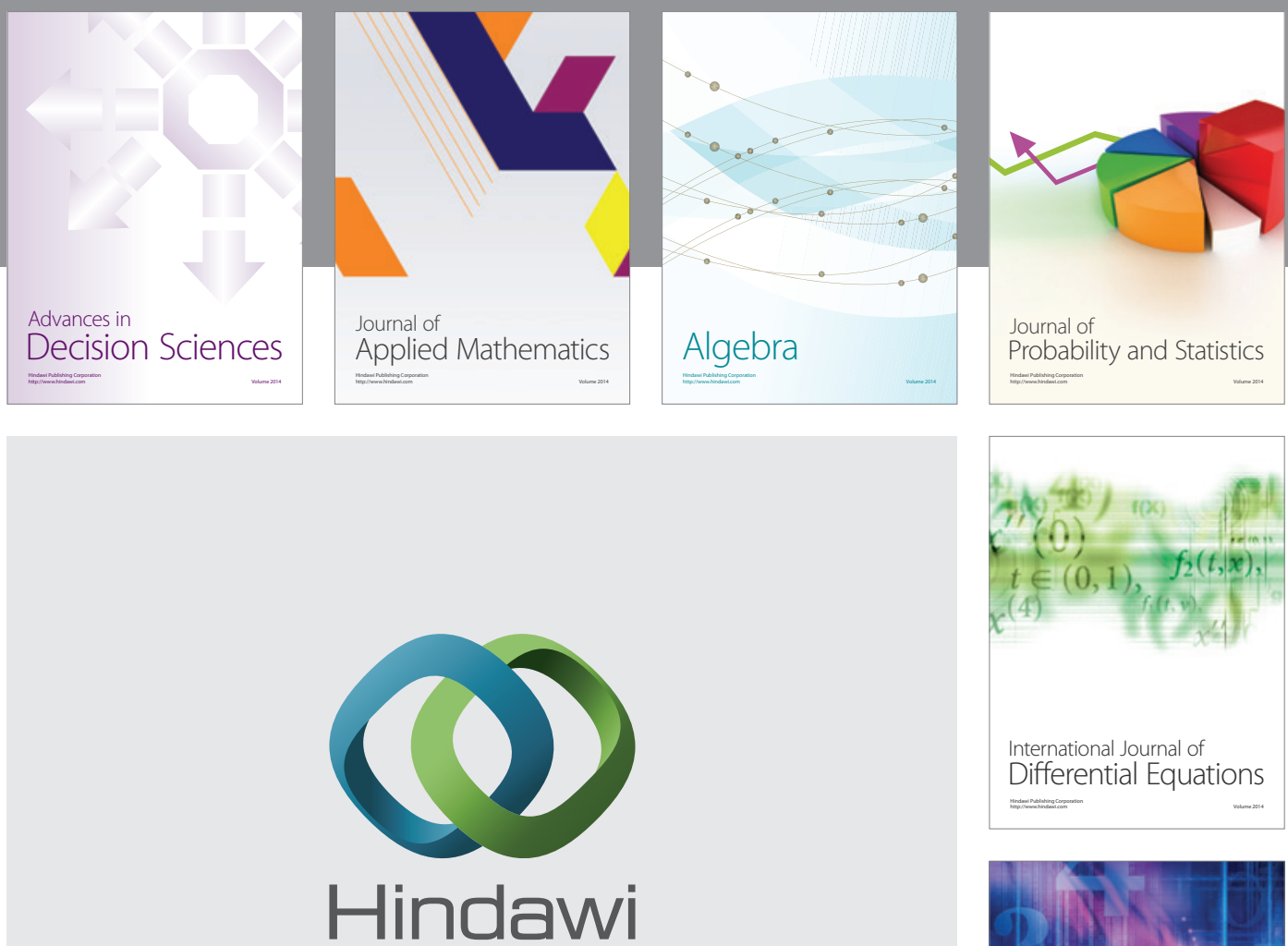

Submit your manuscripts at http://www.hindawi.com
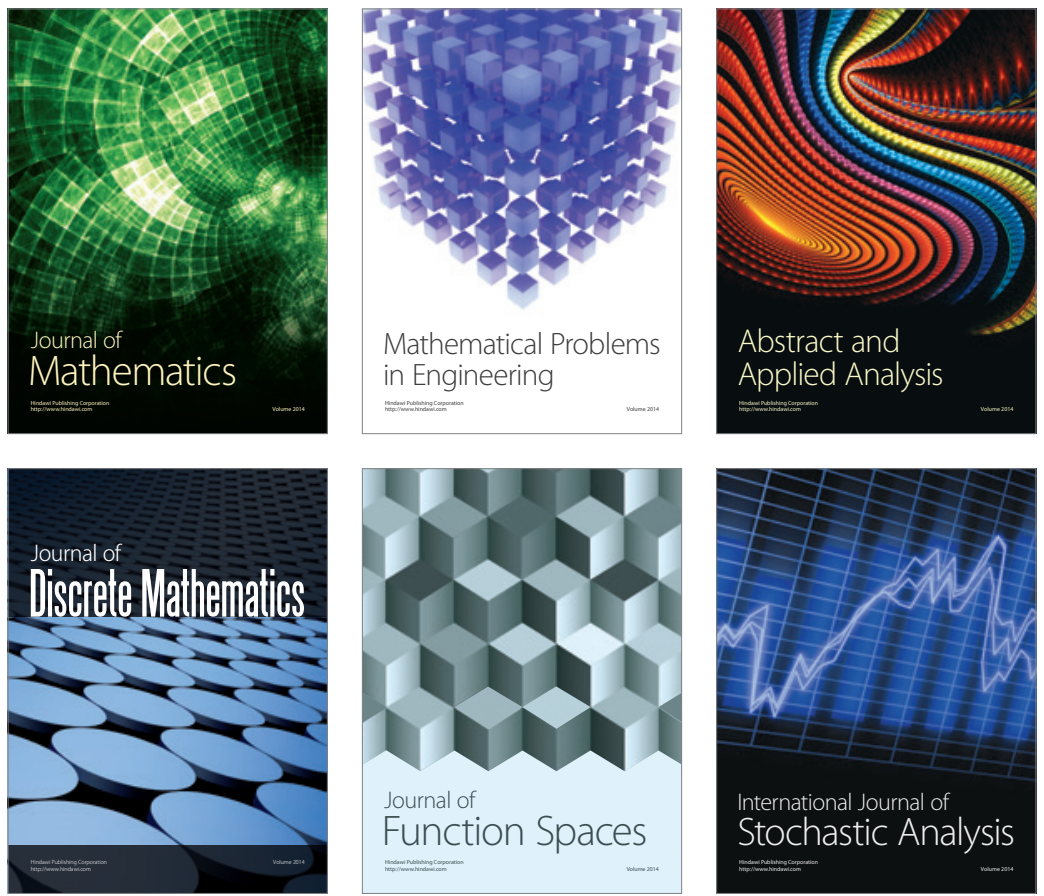

Journal of

Function Spaces

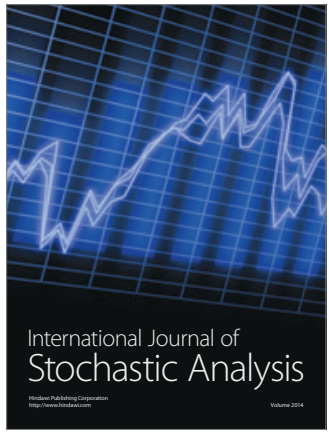

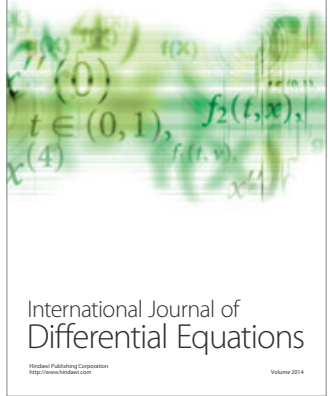
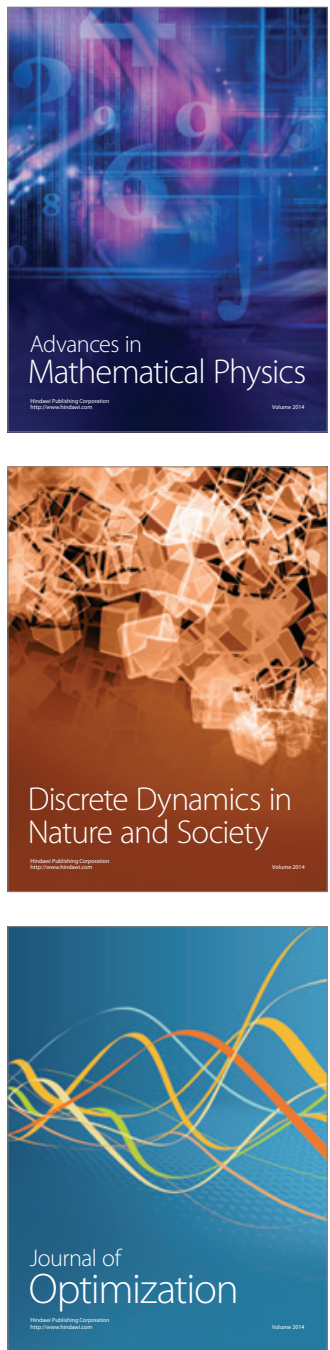